\title{
Physiological and Transcriptome Analysis Reveals the Differences in Nitrate Content Between Lamina and Midrib of Flue-Cured Tobacco
}

\author{
Yuqing Feng \\ Henan Agricultural University \\ Yuanyuan Zhao \\ Henan Agricultural University \\ Yafei Li \\ Henan Agricultural University \\ Jun Zhou \\ Shanghai Tobacco Group Co., Ltd
Yujing Li
Henan Agricultural University
Hongzhi Shi ( $\square$ shihongzhi88@163.com)
Henan Agricultural University

\section{Research Article}

Keywords: flue-cured tobacco, lamina, midrib, nitrate transporter, nitrate accumulation

Posted Date: April 28th, 2021

DOI: https://doi.org/10.21203/rs.3.rs-436881/v1

License: (c) (i) This work is licensed under a Creative Commons Attribution 4.0 International License. Read Full License

Version of Record: A version of this preprint was published at Scientific Reports on February 21st, 2022. See the published version at https://doi.org/10.1038/s41598-022-07011-y. 


\section{Abstract}

Nitrate is an important precursor of tobacco-specific nitrosamines (TSNAs) and remarkable difference in nitrate accumulation between lamina and midrib of flue-cured tobacco has long been observed. However, the physiological and molecular mechanisms underpinning this difference remain poorly understood. In this study, physiological and genetic factors impacting nitrate accumulation were identified in pot experiments using flue-cured tobacco K326 with contrasting nitrate content between lamina and midrib. The results showed that three times higher of $\mathrm{NO}_{3}-\mathrm{N}_{\text {content }}$ was observed in midrib than that in lamina, along with lower pigment, $\mathrm{NH}_{4}-\mathrm{N}$ content, NRA, SSA and GSA in midrib. Transcriptome analysis revealed that expression of genes involved in porphyrin and chlorophyll metabolism, carotenoid biosynthesis, photosynthesis-antenna proteins, photosynthesis, carbon fixation in photosynthetic organisms, starch and sucrose metabolism, nitrogen metabolism and biosynthesis of amino acids were significantly lower in midrib than in lamina. qRTPCR results showed that the expression level of nitrate transporter genes LOC107782967, LOC107806749, LOC107775674, LOC107829632, LOC107799198, LOC107768465 decreased by 2.74, 1.81, 49.5, 3.5, 2.64 and 2.96 folds while LOC107789301 increased by 8.23 folds in midrib but not in lamina. Reduced chlorophyll content might result in low carbohydrate formation which is the source of energy and carbon skeleton supply, then the low capacity of nitrogen reduction, assimilation and transportation, and the poor ability of nitrate reallocation but high capacity of accumulation might lead to nitrate accumulation in midrib. The results laid the foundation for reducing nitrate content and TSNA formation in tobacco midribs and their products.

\section{Introduction}

Tobacco is an industrial crop which is widely grown throughout the world. Tobacco leaf consists of lamina and midrib, with midrib accounting for about $25 \%$ 30\% of the leaf weight. Not only lamina, but also midrib is widely used as raw materials for cigarette production through the making of reconstituted tobacco sheet or midrib cut. Therefore, tobacco midribs have great value when scientifically processed. Midrib has lower tar level, so it plays a significant part in reducing the hazards of cigarettes. The usage of midrib is also benificial to cost cutting, thus improving the use efficiency of tobacco raw materials. However, the disadvantage of midrib is also obvious, among which is substantial higher levels of nitrate content and subsequent higher formation and accumulation of tobacco-specific nitrosamines (TSNAs) [1-2] than that in lamina. Nitrate content in midrib of cured tobacco leaf is usually more than 10 times higher than that in lamina of the same cured leaf[1].

TSNA is prone to induce malignant tumors in animals and was classified as the first class carcinogen by the International Agency for Research on Cancer[3]. It is well recognized that nitrate is an important precursor of tobacco-specific nitrosamines (TSNAs). Nitrate may easily be reduced to nitrite by microbial activity during leaf curing[4] or produce gaseous NOx during leaf storage under warm or hot conditions[5], and the subsequent nitrosation of tobacco alkaloids by these nitrosating agents may lead to much increased lecvels of TSNA formation and accumulation in midrib[1]. Therefore, the reduction of nitrate content is a key for reducing TSNA formation, and the investigation of the mechanisms of nitrate accumulation in midrib is essential, so as to lay the foundation for reducing nitrate content and TSNA formation in tobacco midribs and their products.

Nitrate (NO3-) is one of the main sources of nitrogen absorption by plants, which will accumulate to a large extent in plant cell vacuoles if not reduced, reused or transported into the cytoplasm[6]. Once absorbed by root cells, a larger proportion is transferred to the shoot, where it is rapidly turned into nitrite by nitrate reductase (NR) and nitrite reductase (NiR), and subsequently incorporated into glutamine by glutamine synthetase (GS), which is metabolized to glutamate (Glu) and glutamine (GIn) by GIn synthetase (GS) and Glu synthase (GOGAT), respectively [7-8].

Carbon metabolism is highly correlated with nitrogen metabolism in plants. $\mathrm{N}$ assimilation requires both energy and organic carbon (C) which are provided by photosynthesis[7,9]. A previous study has demonstrated that the lowering of pigment content, carbon fixation and nitrogen assimilation were the main causes of nitrate accumulation in burley tobacco[10]. Moreover, some genes and transcription factors involved in nitrate transport, signaling, and use efficiency can affect the 
content of nitrate. Four protein families are known to be involved in nitrate uptake, distribution or storage: the Nitrate Transporter 1/Peptide Transporter (NPF) family, the Nitrate Transporter 2 (NRT2) family, the Chloride Channel (CLC) family, and the Slow Anion Associated Channel Homolog (SLC/SLAH) family[8]. AtNPF6.3 (also known as CHLORATE RESISTANT 1, CHL1, or NRT1.1) was the first dual-affinity nitrate transporter and also founctions as a nitrate sensor[11]. OsNRT1.1B/OsNPF6.5 also operates as a dual-affinity nitrate transporter and mediates nitrate uptake and root-to-shoot transport[12]. AtNPF7.3/NRT1.5 modulates xylem loading of nitrate in root pericycle cells[13-14]. AtNPF7.2/NRT1.8 is chiefly expressed in xylem parenchyma cells and more nitrate is found in xylem sap in npf7.2 mutants[13]. Interestingly, the functions of NPF7.2 and NPF7.3 are antagonistic, and expressions of NPF7.2 and NPF7.3 are inversely regulated upon stress treatments[12,15]. AtNPF5.11, AtNPF5.12, and AtNPF5.16, localized in tonoplast, were proposed to mediate nitrate efflux from vacuoles and to regulate nitrate distribution between roots and shoots[16]. Moreover, AtNPF6.2/NRT1.4 is predominantly expressed in the petiole and midrib of leaves[17]. Compared with the wild type, less nitrate accumulates in the petiole, but more nitrate is detected in the leaf blade of npf6.2 mutants, indicatting that NPF6.2 participates in nitrate storage of the petiole. NLPs have been suggested to be involved in mediating the early $\mathrm{N}$ response. For instance, transcription factor NIN-LIKE PROTEIN 7 (NLP7) was identified as a primary regulator in nitrate response in Arabidopsis and regulates the expression of several nitrate-responsive genes including NITRATE REDUCTASE 1 (NIA1), NIA2, NRT2.1, and NRT2.2[18-19]. Furthermore, a study showed OsNLP4 transactivats the NRE motif at the promoter of OsNiR encoding nitrite reductase that is a key enzyme determining nitrogen assimilation in rice[20]. In addition to the genes above, other genes which mediate, for example, nitrate signalling and transcription factors also play an essential role in nitrate metabolism[2122].

Recent years, the midrib is also widely used in cigarette production. However, the nitrate content of midrib is markedly higher than that of lamina. Shi et al[10]compared the differences in carbohydrate and nitrate accumulation of flue-cured tobacco and burley tobacco, while inclusive studies about lamina and midrib have never been reported. The aims of this study was to identify the physiological and transcriptome differences between the lamina and midrib, so as to reveal the mechanism of nitrate accumulation in midrib. Significant findings were obtained that would provide insight into the difference in carbon and nitrogen metabolism and valuable gene resources that might explain the reason why midrib had higher nitrate content.

\section{Materials And Methods}

\section{Plant material and study design}

Pot experiments were conducted on substrate culture in greenhouse that maintained a temperature of $25 \pm 2 \circ \mathrm{C}$, average photosynthetic photon flux density of $400 \mu \mathrm{mol} . \mathrm{m}-2 . \mathrm{s}-1$ and relative humidity $80 \%$. The flue-cured tobacco variety $\mathrm{K} 326$ were used in this study. Seeds were sterilized with $2 \%(\mathrm{v} / \mathrm{v})$ sodium hypochlorite for 5 min twice and then were sown in a floating system. Forty days after sowing, seedlings were transplanted in $7.1 \mathrm{~cm} \times 7.8 \mathrm{~cm}$ (diameter $\times$ depth) plastic pots and cultivated with Hoagland solution. Laminas and midribs were collected separately 15 days after seedlings being transplanted. Half of the samples were frozen in liquid nitrogen and stored in a freezer at $-80 \circ \mathrm{C}$, while the other half were deactivated at $105^{\circ} \mathrm{C}$ for $20 \mathrm{~min}$ and then dried at $60^{\circ} \mathrm{C}$ for $48 \mathrm{~h}$. Frozen samples were used for transcriptome analysis, enzyme activity determination, soluble protein and NH4-N content investigation. Dried samples were used for determination of nitrate content. Every treatment had three biological replicates. The K326 seeds used in this study were provided by Yunnan Tobacco Company and the collection of the plant material complied with relevant institutional, national and international guidelines and legislation.

\section{Assays of SSA, NRA and GSA}

Frozen samples were powdered with liquid N2. The activities of SS, NR and GS were determined using SS, NR and GS microdetermination kits (Suzhou Comin Biotechnology Co., Ltd, Jiangsu, China), respectively.

\section{Measurement of pigment content, nitrate, soluble protein and NH4-N content}

Nitrate content was determined by the method described by Cataldo[34]. Samples was frozen in liquid N2 and used to investigate the pigment content and soluble protein content according to Zou [35]. About $0.5 \mathrm{~g}$ of each samples was frozen in liquid N2 and used to investigate the NH4-N content according to Fan[36]. 


\section{RNA Extraction, Preparation of cDNA Library, and Sequencing}

Total RNA was extracted using the mirVana miRNA Isolation Kit (Ambion, Waltham, MA, USA) following the manufacturer's protocol. RNA integrity was evaluated using the Agilent 2100 Bioanalyzer (Agilent Technologies, Santa Clara, CA, USA). The samples with RNA Integrity Number (RIN) $\geq 7$ were subjected to the subsequent analysis. The libraries were constructed using TruSeq Stranded mRNA LTSample Prep Kit (Illumina, San Diego, CA, USA) according to the manufacturer's instructions. These libraries were then sequenced on the Illumina sequencing platform (HiSeqTM 2500 or Illumina HiSeq X Ten) and $125 \mathrm{bp} / 150 \mathrm{bp}$ paired-end reads were generated. Quality control was assessed on the remaining reads using NGS QC Toolkit[37]. After removing low quality date, the clean reads were mapped to reference P. trichocarpa genome (ftp://ftp.solgenomics.net/genomes/Nicotiana_tabacum/assembly/Ntab-K326_AWOJ-SS.fa.gz) using bowtie2 or Tophat (http://tophat.cbcb.umd.edu/)[38-39].

\section{RNA-Seq Analysis, GO and KEGG Pathway Enrichment Analysis of Differentially Expressed Genes (DEGs)}

Transcript profiles of RNA-seq date were analyzed by calculating the read fragments per kilobase per million mapped reads (FPKM). FPKM value of each gene was calculated using cufflinks, and the read counts of each gene were obtained by htseq-count[40-41]. DEGs were identified using the DESeq (2012) functions estimate Size Factors and nbinomTest[42]. Pvalue $<0.05$ and fold change $>2$ or fold change $<0.5$ was set as the threshold for significantly differential expression. Gene function was annotated based on databases of NR (NCBI non-redundant protein sequences), KOG (Clusters of Orthologous Groups of proteins)[43 ], Swiss-Prot (A manually annotated and reviewed protein sequence database)[44 ], KO (KEGG Ortholog database)[45 ], GO (Gene Ontology)[46]. GO enrichment and KEGG pathway enrichment analysis of DEGs were, respectively, achieved using $\mathrm{R}$ based on the hypergeometric distribution. Heatmaps analysis of DEGs was generated with $\mathrm{R}$ (3.4.1 version) (Lucent Technologies, Murray Hill, NJ, USA) pheatmap package[47].

\section{Gene expression analysis by qRT-PCR}

Expression of eight genes related to nitrogen metabolism were observed. qRT-PCR was

performed using Light Real-time PCR Instrument (7900HT FAST, ABI). Reactions were incubated in a 384-well optical plate (Roche, Basel, Swiss) at $50.0^{\circ} \mathrm{C}$ for $2 \mathrm{~min}, 95^{\circ} \mathrm{C}$ for $10 \mathrm{~min}$, followed by 40 cycles of $95^{\circ} \mathrm{C}$ for $15 \mathrm{~s}, 60^{\circ} \mathrm{C}$ for $60 \mathrm{~s}$. TKF and TKR were used as the endogenous control (Tables 2). The expression levels of mRNAs were normalized and calculated using the 2- $\Delta \Delta$ Ct method[48].

\section{Statistical Analysis}

Figures were processed using GraphPad Prism (v. 8.0.1, GraphPad Software Inc., CA, USA) and correlation analysis and variance between treatments were all processed using SPSS 20.0 (IBM, Palo Alto, CA, USA). For comparison between two data sets, a Student's $t$ test was used. ${ }^{\star} \mathrm{P}<0.05,{ }^{\star}{ }^{\mathrm{P}}<0.01$ were considered statistically signifcant. All presented data is mean of three biological replicates $(n=3)$.

\section{Results}

\section{Differences in NO3-N and total nitrogen between lamina and midrib}

Nitrogen contents were different between lamina and midrib (Fig.1a-d). The NO2-N and total nitrogen content in lamina were higher than those in midrib while the NO3-N content and the ratio of NO3-N/total nitrogen content (TN) in midrib were significantly higher than those in lamina, which might be due to the weak ability of nitrogen reutilization, leading to nitrate accumulation in midrib.

\section{Differences in Enzymes Activities Correlated with Nitrogen Metabolism}

NR and GS both play important roles in the process of nitrogen metabolism. In order to explore whether the key enzymes involved in nitrogen metabolism were the key factors impacting nitrate accumulation, we measured the activities of NR, GS and their reaction products in lamina and midrib (Fig.2a-b). It is noteworthy that the nitrate reductase activity (NRA) and glutamine synthetase activity (GSA) were lower in midrib than in lamina. In addition, NH4-N and soluble protein content in midrib were dramatically lower than that in lamina (Fig.2c-d), indicating that the ability of nitrate reduction and assimilation in lamina was higher than midrib.

Differences in pigment content and enzymes activities correlated with carbon metabolism between lamina and midrib 
The results showed that pigment, chlorophyll a, chlorophyll b, carotene contents and enzymes activities correlated with carbon and nitrogen metabolism between lamina and midrib (Fig.3a-d). Chlorophyll a content, chlorophyll b and carotenoid contents were significantly lower in midrib than those in lamina. Also, SSA was always lower in midrib than that in lamina (Fig.3d). Lower pigment content may have an influence on carbon fixation and lead to low carbohydrate accumulation in midrib.

\section{Quality control and sequence alignment}

After filtering the raw reads, a high rate of clean reads from each sample was achieved. In all, the mapping rates of all the samples to the reference genome were above $93 \%$, the GC content of all samples was stable with the distribution ranging from 43.16 44.09\% and the QC30 value of all sample was above 91\% (Table 1), implying successful library construction and RNA sequencing.

\section{Quality control, gene expression and correlation analysis between samples}

As shown in Fig.4a, the FPKM expression levels for each sample were calculated. In addition, Pearson's correlation coefficient and principle component analysis (PCA) of the data profiles from all 6 samples revealed high correlation among all the samples (Fig.4b-C). As shown in Fig.4b, the square of PCC was distributed between 0.88 and 0.98 . These analysis demonstrated that the sequencing data in this study was adequately representative and valid.

\section{Differentially expressed gene selection, Go enrichment and KEGG pathway analysis of differentially expressed genes}

The differentially expressed genes (DEGs) were achieved. In the process of DEGs screening, fold change (FC) $>2$ or $F C<0.5$, p-value $<0.05$, was used as threshold to determine the significance of gene expression differences. A total of $7560 \mathrm{DEGs}$ (3446 up-regulated and 4114 down-regulated) were identified between lamina and midrib groups (Fig.5a). And the volcano of differentially expressed genes between lamina and midrib was achieved (Fig.5b).

The DEGs in lamina vs midrib were further analyzed using GO and KEGG analyses (Fig.5c-f). In detail, the down-regulated DEGs in lamina vs midrib were significantly enriched in photosynthesis - antenna proteins, photosynthesis, porphyrin and chlorophyll metabolism, carbon fixation in photosynthetic organisms, carotenoid biosynthesis, photosystem II and photosystem I. The up-regulated genes were mostly involved in cell wall organization, phenylpropanoid biosynthesis, steroid biosynthesis, xyloglucan metabolic process, pentose and glucuronate interconversions and transporter activity.

\section{Comparative analysis of differentially expressed genes correlated with carbon and nitrogen metabolism}

Transcriptome sequencing technology provides a large amount of information regarding the DEGs that are involved in specific biological responses. Fig.6 show that porphyrin and chlorophyll metabolism, carotenoid biosynthesis, photosynthesis-antenna proteins, photosynthesis, carbon fixation in photosynthetic organisms, starch and sucrose metabolism, nitrogen metabolism and biosynthesis of amino acids were lower in midrib than in lamina. In addition, we searched the genes involved in porphyrin and chlorophyll metabolism (LOC107777980, LOC107786828 and LOC107788874) (Fig.6a), carotenoid biosynthesis (LOC107772713, LOC107763949, LOC107763628, and LOC107797654) (Fig.6b), photosynthesis-antenna proteins (LOC107773808, LOC107776229, LOC107778264, LOC107782430, LOC107772663, LOC107773232 and LOC107764358) (Fig.6C), photosynthesis (LOC107763149, LOC107810205, LOC107784985, LOC107766588 and LOC107768924) (Fig.6d), carbon fixation in photosynthetic organisms (LOC107780142, LOC107777241, LOC107771723 and LOC107766567) (Fig.6e), starch and sucrose metabolism (LOC107761864, LOC107825407 and LOC107771409) (Fig.6f), nitrogen metabolism (LOC107768773 and LOC107766022) (Fig.6g) and biosynthesis of amino acids (LOC107785928, LOC107784332, LOC107766022 and LOC107794948) (Fig.6h) were greatly suppressed in midrib. To explore the reason why midrib hold higher nitrate than lamina, we analyzed the differences in gene expression levels of nitrate response, transport and assimilition. The results showed that genes of NLP4, NLP7, NPF2.13, NPF3.1, NPF6.3, NPF7.3, NIA, GS and GOGAT were down-regulated in midrib while genes of NPG1.2 and NPF7.2 were up-regulated in (Fig.6i), which might also be the cause for higher nitrate content in midrib.

\section{Expression levels of genes related to nitrate transport}

Nitrate transporters play an essential role in nitrogen metabolism. Expression level of genes involved in nitrate transporting (LOC107782967, LOC107806749, LOC107775674, LOC107829632, LOC107799198, LOC107768465) was down-regulated while LOC107789301 and LOC107770138 were up-regulated in midrib compared to that in lamina(Fig.7). And the qRT-PCR results showed that the expression patterns of the eight genes were identical to those detected by transcriptome 
sequencing, which confirmed the reliability of RNA-seq data and explained the reason why nitrate content was higher in midrib than that in lamina.

\section{Discussion}

In recently years, the midrib has been widely used in cigarette production in the form of tobacco sheet. However, our study showed that the midrib had higher NO3-N content of more than 3 times than lamina (Fig.1a), which is not beneficial to tobacco safety and harm reduction. One strategy to decrease the content of nitrate is to identify the physiological and molecular mechanisms contributing to nitrate accumulation in midrib. In the studies presented here, pots experiment was employed to study the physiological and transcriptome differences between lamina and midrib. Overall, the present study demonstrated that the expression of genes involved in porphyrin and chlorophyll metabolism, carotenoid biosynthesis, photosynthesis-antenna proteins, photosynthesis, carbon fixation in photosynthetic organisms, starch and sucrose metabolism, nitrogen metabolism and biosynthesis of amino acids were significantly lower in midrib than in lamina (Fig.6a-h).

It has long been recognized that chlorophyll content is used as an indicator of photosynthetic capacity and photosynthesis and $\mathrm{C}$ metabolism functions to provide both energy and $\mathrm{C}$ skeletons for plant growth and $\mathrm{N}$ assimilation[23,7]. Our results showed that the midrib had lower chlophyll a, chlophyll b, carotenoid and SSA than that of lamina (Fig.3a-d). Some key genes related to pigment biosynthetic process and $\mathrm{C}$ metabolism were significantly down-regulated in midrib, including LOC107777980 (MgPME)[24 ], LOC107783891 (CHLI)[25 ], LOC107763283 (hemA) and LOC107783257(CHLP)[26 ], which play crucial roles in chlophyll biosynthesis, LOC107772713 (PSY2)[27-28], which encodes phytoene synthase and controls the carbon flux through the carotenoid biosynthetic pathway, LOC107785687(SPS2) edcodes sucrose-phosphate synthase that plays the role of rate-limiting steps in sucrose synthesis in higher plant[29]. The lower chlorophyll content resulted in a decrease of the chlorophyll a/b binding proteins in midrib. LOC107772663 (LHCb1), which is one of the most abundant chloroplast proteins in plants and mainly function to collect and transfer light energy to photosynthetic reaction centers[30], was significantly repressed in midrib. PSI, PSIl, and photosynthetic electron transport are key components in the photosynthetic pathway. While LOC107810205 (PsbR), LOC107784985(PsaO) and LOC107803171 (petF), which were involved in PSI, PSII, and photosynthetic electron transport were down regulated in midrib. LOC107771723(Rubisco) encodes a key enzyme in the calvin cycle and assimilates atmospheric $\mathrm{CO} 2$ into the biosphere[31]. This is consistent with the physiological differences between lamina and midrib.

NR and GS are two of the most important enzymes in N assimilation [8]. The ammonium taken up by AMTs or derived from nitrate is used to produce a variety of amino acids via the GS/GOGAT cycle [7]. NLP7 is a primary regulator in nitrate response and regulates the expression of several nitrate-responsive genes including NIA1, NIA2, NRT2.1, and NRT2.2[18-19]. And OsNLP4 transactivats the NRE motif at the promoter of OsNiR encoding nitrite reductase in rice[20]. In this study, NLP7 and NLP4 were down-regulated in midrib, which was inconducive to the decrease of nitrate accumulation in midrib. Further investigation of the expression of genes encoding nitrate response, transport and assimilation led to the discovery of nitrate response genes (NPF6.3, NLP4 and NLP7), nitrate transporters (NPF2.13, NPF3.1, NPF7.3, NPF1.2 and NPF7.2) and nitrate assimilation genes (NIA, GS and GOGAT) with contrasting transcriptional responses in lamina and midrib. And our results showed that midrib was lower in NR activity, GS activity, NH4-N and soluble protein content while higher in NO3-N and NO3$\mathrm{N} / \mathrm{TN}$ than midrib, suggesting that midrib might retain a weaker capacity of nitrate assimilation. NPF7.3/NRT1.5 mediates efflux of NO3-to the xylem vessels, whereas NPF7.2/NRT1.8 performs the opposite function and retrieves NO3-from the xylem sap into xylem parenchyma cells $[32,14]$. NPF2.13 can facilitate outward nitrate transport by phloem loading.

Moreover, NPF1.2 is expressed in the companion cells of the major veins in expanded leaves and involved in diverting rootderived nitrate into phloem in the major vein of mature and expanded leaves[33].qRT-PCR results showed that the nitrate transporter genes LOC107782967, LOC107806749, LOC107775674, LOC107829632, LOC107799198, LOC107768465 were down-regulated while LOC107789301 and LOC107770138 were up-regulated in midrib but not in lamina, indicating that midrib had poor ability in reallocation nitrate transported by roots.

In Conclusion, significant differences were observed in nitrate accumulation between lamina and midrib of flue-cured 
tobacco. Pigment content and SSA in midrib were significantly lower than that in lamina, which resulted in insufficient C skeleton for nitrogen metabolism. Meanwhile, the greater nitrate accumulation was probably conferred by more disadvantageous aspects such as weak nitrogen reduction, weak nitrogen assimilation, poor ability in reallocation and high capacity of accumulating nitrate in midrib than in lamina. The above insights to physiological and molecular basis of carbon and nitrogen differences in lamina and midrib would be helpful for providing direction for decreasing nitrate accumulation in the midrib.

\section{Declarations}

\section{Acknowledgments}

The study was financially supported by Shanghai Tobacco (Group) Corporation Research Program (grant number TP2019C4).

\section{Authors' contributions}

H.S. performed the conception and design of the research. Y.Z. and Y.L. prepared the figures1-7 and tables 1-2. J.Z. and Y.L. interpreted the data. Y.F. drafted the manuscript. H.S. completed the revision of manuscript for important intellectual content. All authors reviewed the final manuscript.

\section{Conflicts of Interest}

The authors declare that they have no conflicts of interest.

\section{Data Availability Statement}

The sequencing data were deposited in National Center of Biotechnology Information database (https://www.ncbi.nlm.nih.gov/bioproject/ PRJNA720776). The datasets used and/or analyzed during the current study are available from the corresponding author on reasonable request.

\section{References}

[1] Shi, H., Wang, R., Bush, L. P., Zhou, J., Yang, H., Fannin, N., \& Bai, R. Changes in TSNA contents during tobacco storage and the effect of temperature and nitrate level on TSNA formation. Journal of agricultural and food chemistry, 61(47), 11588-11594 (2013).

[2] BokelmAn, G. H., Ryan, W. S.. Analyses of bright and burley tobacco laminae and stems[J]. Beiträge zur Tabakforschung International/Contributions to Tobacco Research, 13(1): 29-36 (1985).

[3] WHO. IARC monographs on the evaluation of carcinogenic risks to humans - Smokeless tobacco and some tobaccospecifc Nnitrosamines. 89, 592, http://monographs.iarc.fr/ENG/Monographs/vol89/mono89.pdf (2007).

[4] Bush, L.P., Hamilton, J.L., Davis, D.L. Chemical quality of burley tobacco modified by curing regime. Tob Chem Res Con, 33: 10 (1979).

[5] Wang, Jun, et al. "Nitrate and nitrite promote formation of tobacco-specific nitrosamines via nitrogen oxides intermediates during postcured storage under warm temperature." Journal of Chemistry 2017 (2017).

[6] Reddy, K. S., \& Menary, R. C. (1990). Nitrate reductase and nitrate accumulation in relation to nitrate toxicity in Boronia megastigma. Physiol. Plant., 78(3), 430-434 (1990).

[7] Xu, G., Fan, X., \& Miller, A. J. Plant nitrogen assimilation and use efficiency. Annual review of plant biology, 63, 153-182 (2012).

[8] Tegeder, M., \& Masclaux--Daubresse, C. Source and sink mechanisms of nitrogen transport and use. New phytologist, 217(1), 35-53 (2018).

[9] Nunes-Nesi, A., Fernie, A. R., \& Stitt, M. Metabolic and signaling aspects underpinning the regulation of plant carbon nitrogen interactions. Molecular plant, 3(6), 973-996 (2010). 
[10] Li, Y., Yang, H., Chang, D., Lin, S., Feng, Y., Li, J., \& Shi, H. Biochemical, physiological and transcriptomic comparison between burley and flue-cured tobacco seedlings in relation to carbohydrates and nitrate content. Molecules, 22(12), 2126 (2017).

[11] Ho, C. H., Lin, S. H., Hu, H. C., \& Tsay, Y. F. CHL1 functions as a nitrate sensor in plants. Cell, 138(6), 1184-1194 (2009).

[12] Hu, Bin, et al. "Variation in NRT1. 1B contributes to nitrate-use divergence between rice subspecies." Nature Genetics 47(7): 834 (2015).

[13] Li, Jian-Yong, et al. "The Arabidopsis nitrate transporter NRT1. 8 functions in nitrate removal from the xylem sap and mediates cadmium tolerance." The Plant Cell 22(5): 1633-1646 (2010).

[14] Lin, Shan-Hua, et al. "Mutation of the Arabidopsis NRT1. 5 nitrate transporter causes defective root-to-shoot nitrate transport." The Plant Cell, 20(9): 2514-2528 (2008):

[15] Zhang, G. B., Yi, H. Y., \& Gong, J. M. The Arabidopsis ethylene/jasmonic acid-NRT signaling module coordinates nitrate reallocation and the trade-off between growth and environmental adaptation. The Plant Cell, 26(10), 3984-3998 (2014).

[16] He, Y. N., Peng, J. S., Cai, Y., Liu, D. F., Guan, Y., Yi, H. Y., \& Gong, J. M. Tonoplast-localized nitrate uptake transporters involved in vacuolar nitrate efflux and reallocation in Arabidopsis. Scientific reports, 7(1), 1-9 (2017).

[17] Chiu, C. C., Lin, C. S., Hsia, A. P., Su, R. C., Lin, H. L., \& Tsay, Y. F. Mutation of a nitrate transporter, AtNRT1: 4, results in a reduced petiole nitrate content and altered leaf development. Plant and Cell Physiology, 45(9), 1139-1148 (2004).

[18]Marchive, Chloéé, et al. "Nuclear retention of the transcription factor NLP7 orchestrates the early response to nitrate in plants." Nature communications 4(1): 1-9 (2013).

[19] O'Brien, J. A., Vega, A., Bouguyon, E., Krouk, G., Gojon, A., Coruzzi, G., \& Gutiéérrez, R. A. Nitrate transport, sensing, and responses in plants. Molecular plant, 9(6), 837-856 (2016).

[20] Yu, Jun, et al. "Enhanced OsNLP4--OsNiR cascade confers nitrogen use efficiency by promoting tiller number in rice." Plant biotechnology journal 19(1): 167-176 (2021).

[21] Ge, Min, et al. "The NIN--like protein 5 (ZmNLP5) transcription factor is involved in modulating the nitrogen response in maize." The Plant Journal 102.2: 353-368 (2020).

[22] Liu, Kun-hsiang, et al. "Discovery of nitrate--CPK--NLP signalling in central nutrient-- growth networks." Nature 545(7654): 311-316 (2017).

[23] Nunes-Nesi, A., Fernie, A. R., \& Stitt, M. Metabolic and signaling aspects underpinning the regulation of plant carbon nitrogen interactions. Molecular plant, 3(6), 973-996 (2010).

[24] Kong, Weiyi, et al. "The catalytic subunit of magnesium-protoporphyrin IX monomethyl ester cyclase forms a chloroplast complex to regulate chlorophyll biosynthesis in rice." Plant molecular biology 92(1): 177-191(2016).

[25] Masuda, T. Recent overview of the Mg branch of the tetrapyrrole biosynthesis leading to chlorophylls. Photosynthesis Research, 96(2), 121-143 (2008).

[26] Zeng, Z., Q., et al. "OsHemA gene, encoding glutamyl-tRNA reductase (GluTR) is essential for chlorophyll biosynthesis in rice (Oryza sativa)." Journal of Integrative Agriculture 19(3): 612-623 (2020).

[27] Just, B. J., Santos, C. A. F., Fonseca, M. E. N., Boiteux, L. S., Oloizia, B. B., \& Simon, P. W. Carotenoid biosynthesis structural genes in carrot (Daucus carota): isolation, sequence-characterization, single nucleotide polymorphism (SNP) markers and genome mapping. Theoretical and Applied Genetics, 114(4), 693-704 (2007).

[28] Maass, D., Arango, J., Wüüst, F., Beyer, P., \& Welsch, R. Carotenoid crystal formation in Arabidopsis and carrot roots caused by increased phytoene synthase protein levels. PloS one, 4(7), e6373 (2009).

[29] Volkert, Kathrin, et al. "Loss of the two major leaf isoforms of sucrose-phosphate synthase in Arabidopsis thaliana limits sucrose synthesis and nocturnal starch degradation but does not alter carbon partitioning during photosynthesis." Journal of experimental botany 65.18: 5217-5229 (2014).

[30] Xia, Y., S., et al. "Allelic variations of a light harvesting chlorophyll a/b-binding protein gene (Lhcb1) associated with agronomic traits in barley." PLoS One 7(5): e37573 (2012).

[31] Mi, C., et al. Cloning of RuBisCo subunits genes rbcL and rbcS from winter rapeseed (Brassica rapa) and their 
expression under drought stress. Acta Agronomica Sinica, 44(12), 1882-1890 (2018).

[32] Chen, C. Z., Lv, X. F., Li, J. Y., Yi, H. Y., \& Gong, J. M. Arabidopsis NRT1. 5 is another essential component in the regulation of nitrate reallocation and stress tolerance. Plant Physiology, 159(4), 1582-1590 (2012).

[33] Wang, Y. Y., Cheng, Y. H., Chen, K. E., \& Tsay, Y. F. Nitrate transport, signaling, and use efficiency. Annual Review of Plant Biology, 69, 85-122 (2018).

[34] Cataldo, D. A., Haroon, M., Schrader, L. E. \& Youngs, V. L. Rapid cplorimetric deternination of nitrate in plant-tissure by nitration of salicylic acid. Commun. Soil Sci. Plan. 6, 71-80 (1975).

[35] Zou, Q. Guide of plant physiological experiments[M]. Beijing: China Agriculture Press: 56-59 (2000).

[36] Fan, S. Q., Liang, S. W. Guidance of modern plant physiology[M]. Shanghai: Science Press, 303-306 (1999).

[37] Patel, R. K., \& Jain, M. NGS QC Toolkit: a toolkit for quality control of next generation sequencing data. PloS one, 7(2), e30619 (2012).

[38] Langmead, B., \& Salzberg, S. L. Fast gapped-read alignment with Bowtie 2. Nat. methods, 9(4), 357 (2012).

[39] Kim, D., Pertea, G., Trapnell, C., Pimentel, H., Kelley, R., \& Salzberg, S. L. TopHat2: accurate alignment of transcriptomes in the presence of insertions, deletions and gene fusions. Genome Biol., 14(4), 1-13 (2013).

[40] Trapnell, C. et al. Differential gene and transcript expression analysis of RNA-seq experiments with TopHat and Cufflinks. Nat. Protoc., 7, 562-578 (2012).

[41] Anders, S., Pyl, P. T., \& Huber, W. HTSeq-a Python framework to work with high-throughput sequencing data.

Bioinformatics, 31(2), 166-169 (2015).

[42] Anders, S., \& Huber, W. Differential expression of RNA-Seq data at the gene level-the DESeq package. Heidelberg, Germany: European Molecular Biology Laboratory (EMBL), 10, f1000research (2012).

[43] Koonin, Eugene V., et al. "A comprehensive evolutionary classification of proteins encoded in complete eukaryotic genomes." Genome biology 5.2: 1-28 (2004).

[44] Apweiler, Rolf, et al. "UniProt: the universal protein knowledgebase." Nucleic acids research 32.suppl_1: D115-D119 (2004).

[45] Kanehisa, M., Goto, S., Kawashima, S., Okuno, Y., \& Hattori, M. The KEGG resource for deciphering the genome. Nucleic acids research, 32(suppl_1), D277-D280 (2004).

[46] Ashburner, Michael, et al. "Gene ontology: tool for the unification of biology." Nature genetics 25.1: 25-29 (2000).

[47] Korpelainen, E., Tuimala, J., Somervuo, P., Huss, M., \& Wong, G.. RNA-seq data analysis: a practical approach. CRC Press (2014).

[48] Livak, K. J. \& Schmittgen, T. D. Analysis of relative gene expression data using real-time quantitative PCR and the 22DDCT method. Methods. 25, 402-408 (2001).

\section{Tables}

Table 1. Statistics of sequencing data quality

\begin{tabular}{|llllllll|}
\hline Sample & $\begin{array}{l}\text { Raw } \\
\text { reads }\end{array}$ & $\begin{array}{l}\text { Clean } \\
\text { reads }\end{array}$ & $\begin{array}{l}\text { Total } \\
\text { reads }\end{array}$ & Total mapped & Q20(\%) & Q30(\%) & GC content(\%) \\
\hline K326YP_1 & 48864160 & 48408636 & 48408636 & $45265003(93.51 \%)$ & 96.87 & 91.7 & 43.74 \\
\hline K326YP_2 & 47517992 & 47096404 & 47096404 & $43990986(93.41 \%)$ & 96.81 & 91.6 & 44.09 \\
\hline K326YP_3 & 52667494 & 52209758 & 52209758 & $48818506(93.5 \%)$ & 96.87 & 91.73 & 43.97 \\
\hline K326ZM_1 & 46260746 & 45909440 & 45909440 & $43168725(94.03 \%)$ & 96.96 & 91.89 & 43.16 \\
\hline K326ZM_2 & 48407794 & 48000324 & 48000324 & $45097971(93.95 \%)$ & 96.85 & 91.66 & 43.24 \\
\hline K326ZM_3 & 50465696 & 50053210 & 50053210 & $47136723(94.17 \%)$ & 97.03 & 92.03 & 43.27 \\
\hline
\end{tabular}


Table 2. The primers used in real-time PCR.

\begin{tabular}{|ll|}
\hline Primer name & Primer sequencel5'-3'0 \\
\hline LOC107782967-TKF & TCAGACATGGGTTCCGTGTG \\
\hline LOC107782967-TKR & GGGGGTCAGCAACATAGCAA \\
\hline LOC107806749-TKF & CAACACGACAGGCAAAGCAG \\
\hline LOC107806749-TKR & CAAATCATCGGCAGCAGCAT \\
\hline LOC107775674-TKF & TGGAGGGCTATGCCTTATGTT \\
\hline LOC107775674-TKR & AAGCACCGAGCAATGGTATGA \\
\hline LOC107829632-TKF & CAGTGGTCGTTGATGGTGATG \\
\hline LOC107829632-TKR & TTGATAGGCTGGCAGGAGGTA \\
\hline LOC107799198-TKF & GTTCCGATTTGTCGTCGTTTC \\
\hline LOC107799198-TKR & GTGGCATTTGCATCATTGGTC \\
\hline LOC107768465-TKF & GGATGAAGGAATGTGGGCTCT \\
\hline LOC107768465-TKR & TCTTCGGTTTCTGGTGTCTCG \\
\hline LOC107789301-TKF & TCCGTGCCAACGAACAAAT \\
\hline LOC107789301-TKR & TCGACTGCAACGCCATCTT \\
\hline LOC107770138-TKF & GGGTTGTCCATGTCTTCCTCA \\
\hline LOC107770138-TKR & TCCAAGTGCCCGTCGTTTA \\
\hline Actin-TKF & CTGAGGTCCTTTTCCAACCA \\
\hline Actin-TKR & TACCCGGGAACATGGTAGAG \\
\hline
\end{tabular}

Figures 
(a)

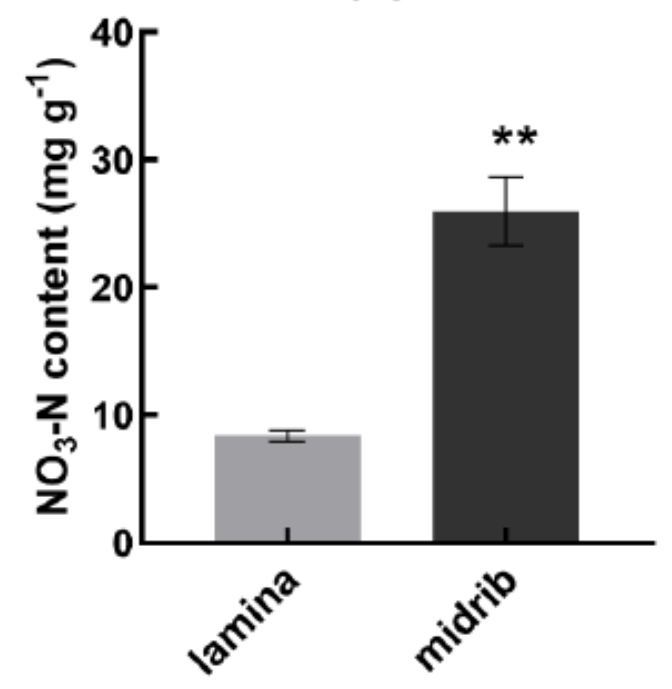

(c)

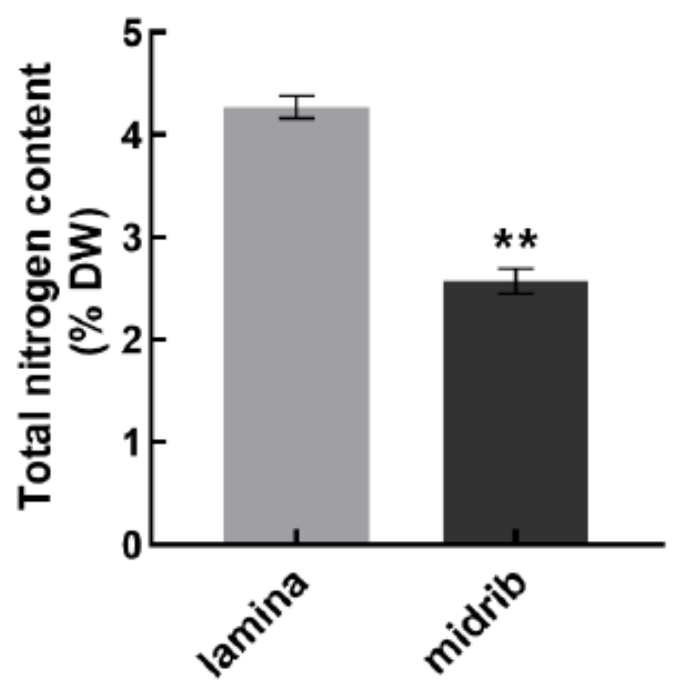

(b)

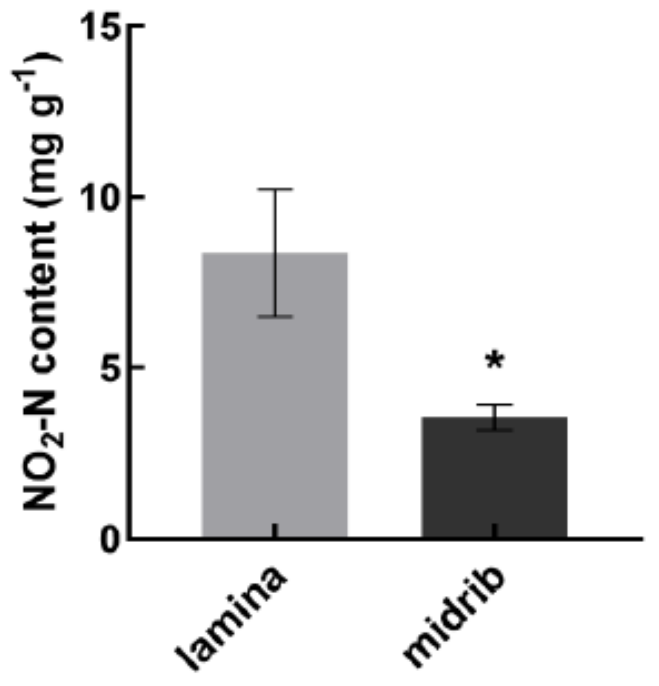

(d)

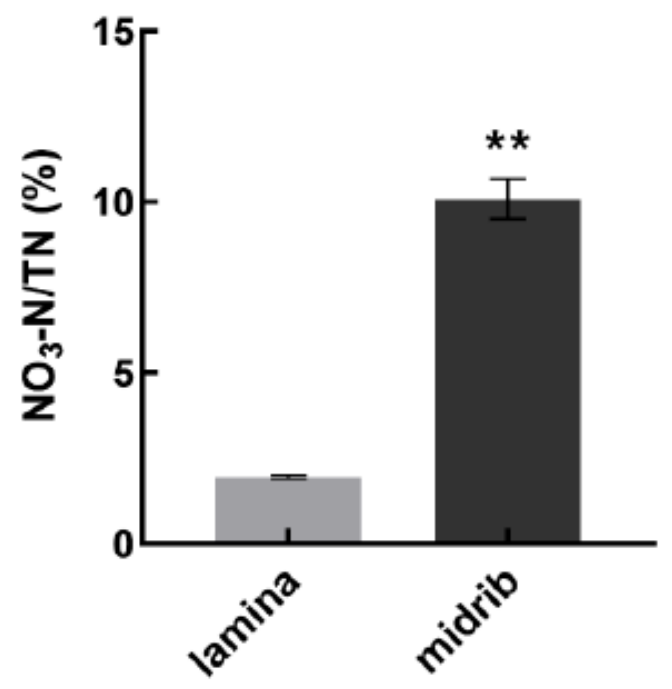

Figure 1

(a) NO3-N content; (b) NO2-N content; (c) total nitrogen content; (d) NO3-N/TN. Symbols ** and * indicates that the significant differences between lamina and midrib at 0.01 and 0.05 . 
(a)

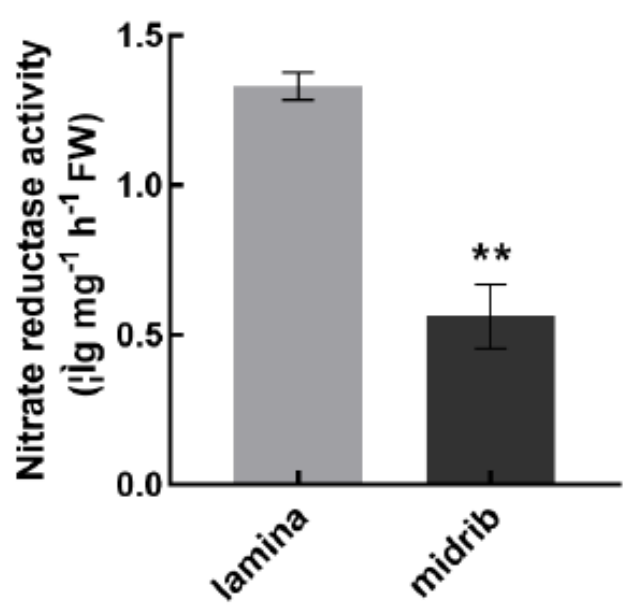

(c)

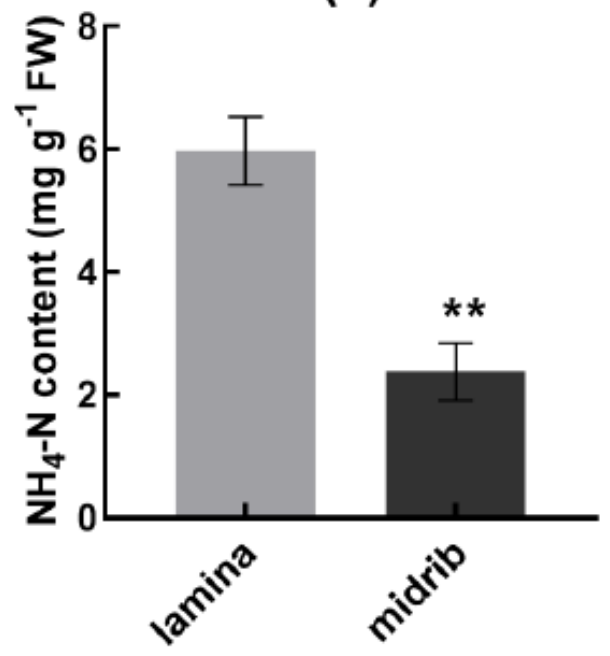

(b)

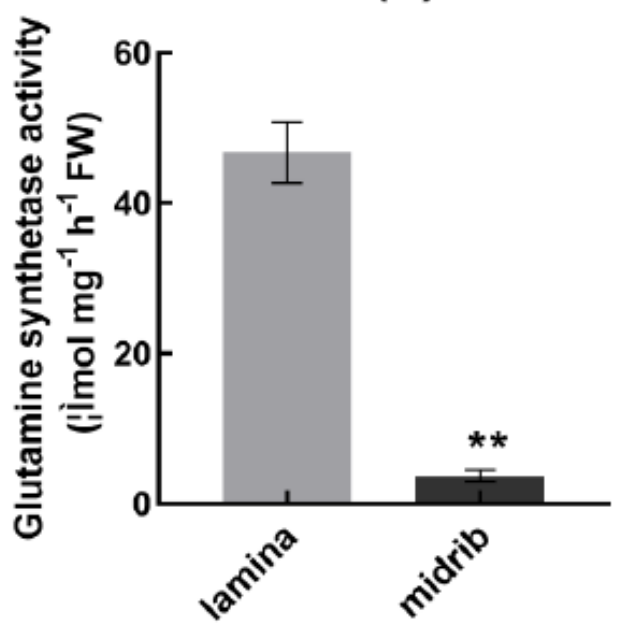

(d)

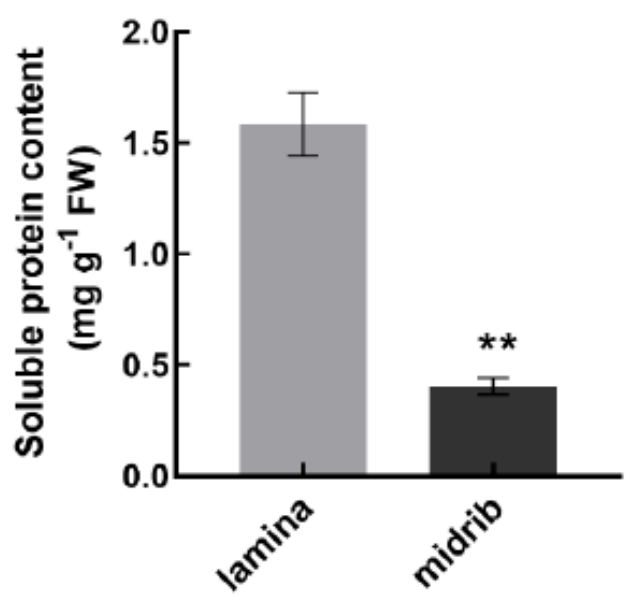

Figure 2

(a) nitrate reductase activity; (b) glutamine synthease activity; (c) NH4-N content; (d) and soluble protein content. Symbols ** indicates that the significant differences between lamina and midrib at 0.01 . 
(a)

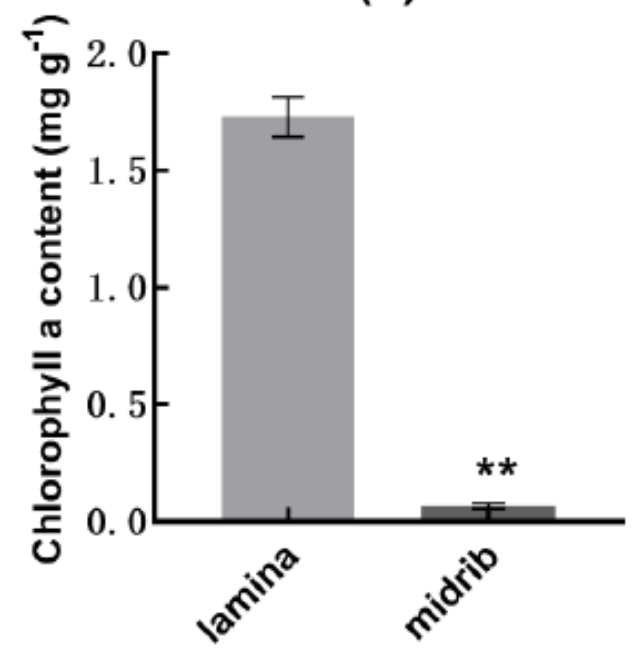

(c)

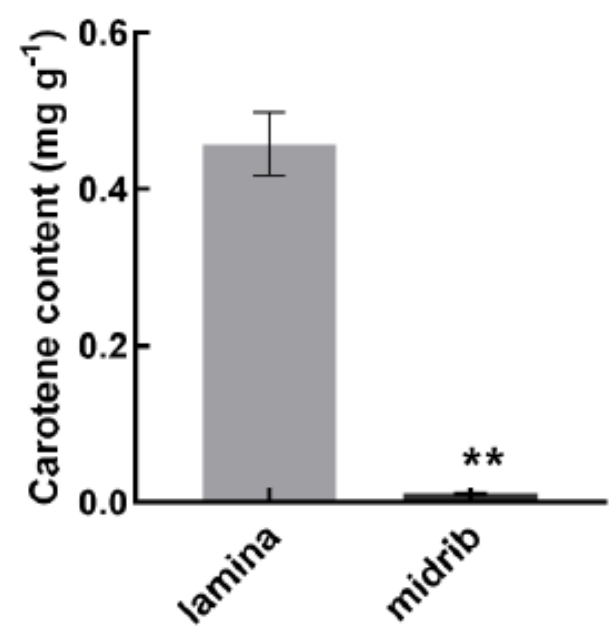

(b)

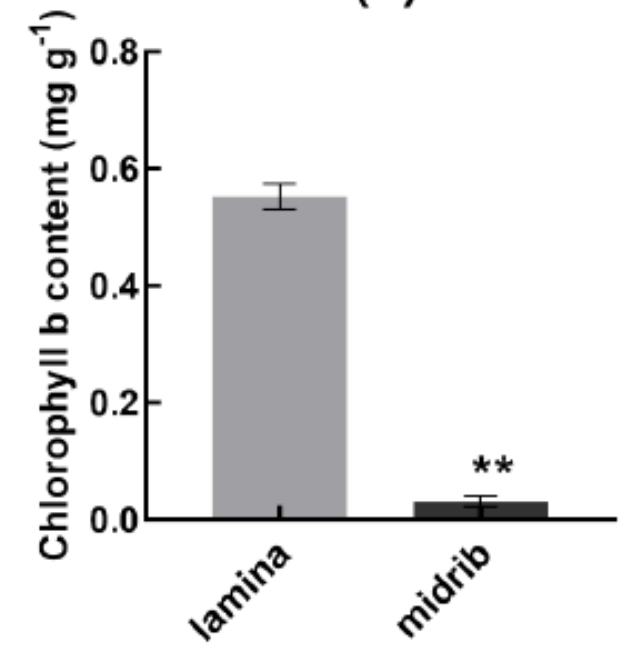

(d)

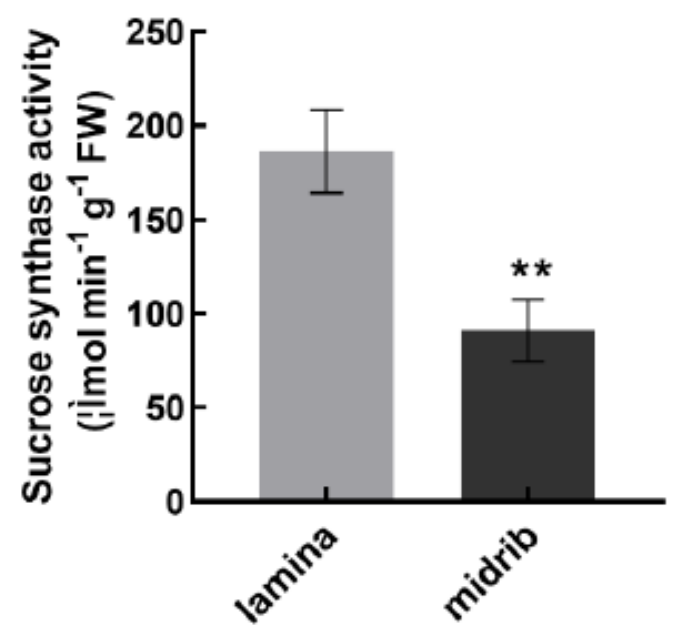

Figure 3

(a) chlorophyll a; (b) chlorophyll b; (c) carotene content; (d) sucrose synthetase. Symbols ** indicates that the significant differences between lamina and midrib at 0.01 . 
(a)

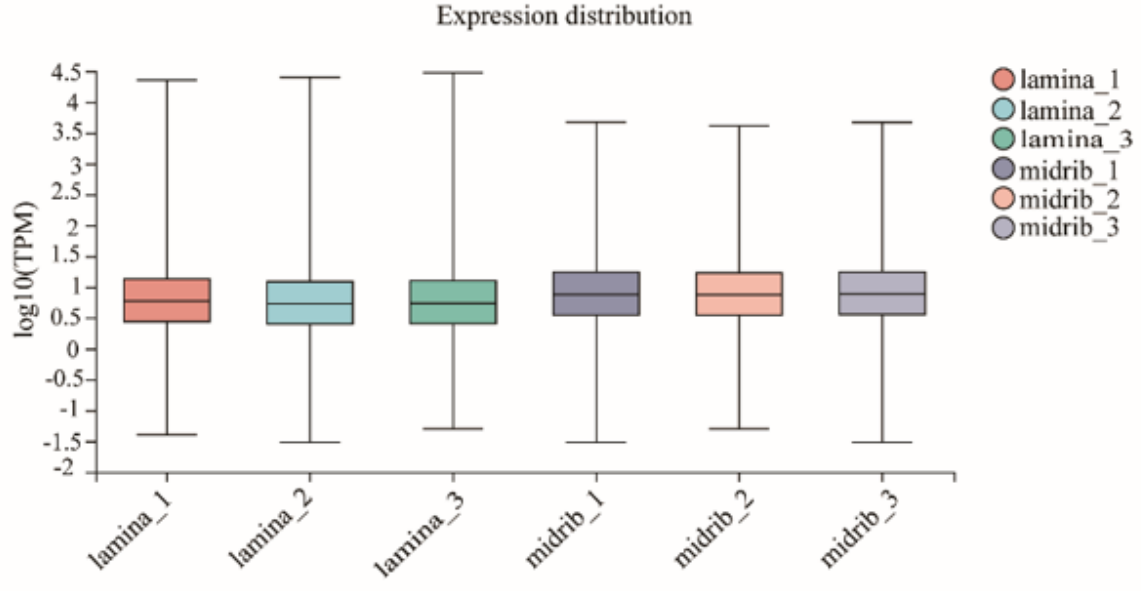

(b)

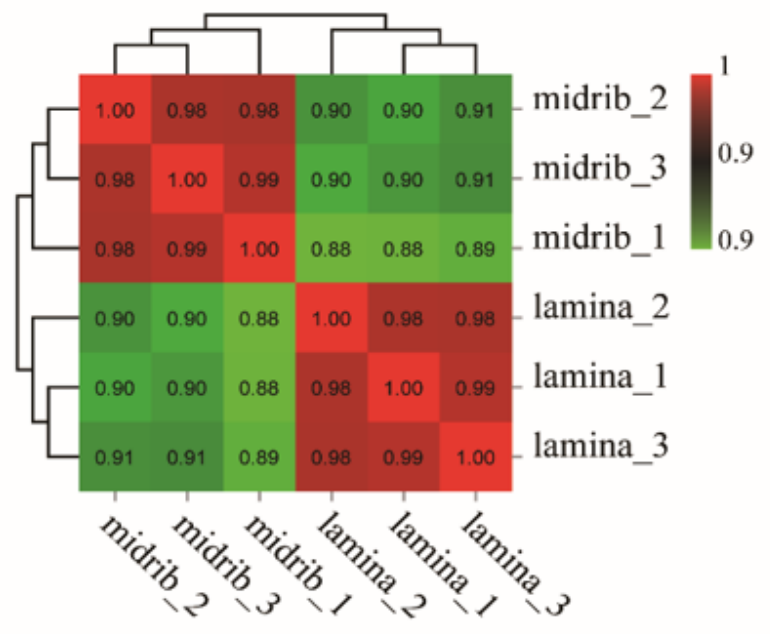

(c)

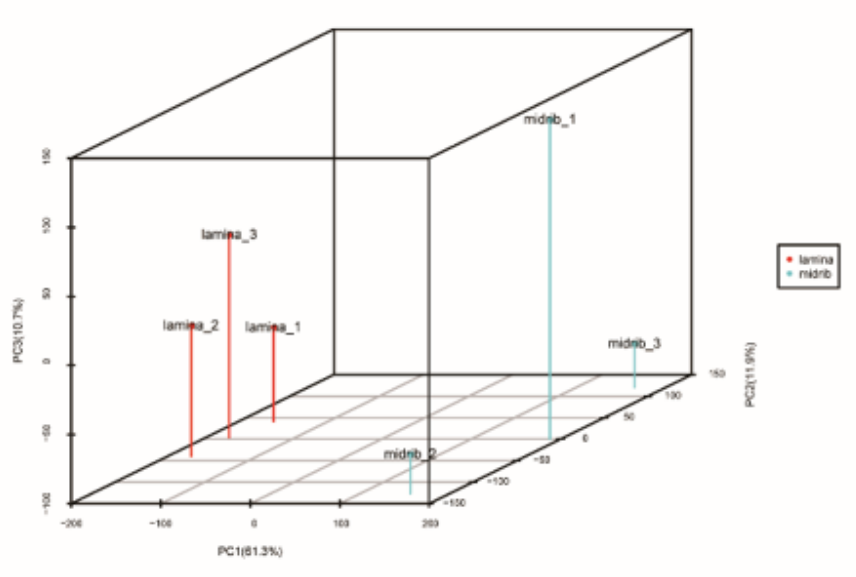

Figure 4

(a) Gene expression levels in samples. (b) Heatmap of correlation between samples. (c) The results of principal component analysis. 
(a)

Statistic of Differently Expression gene

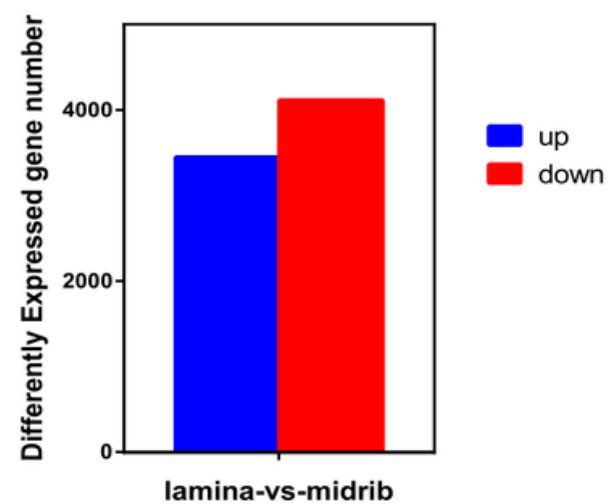

(b)

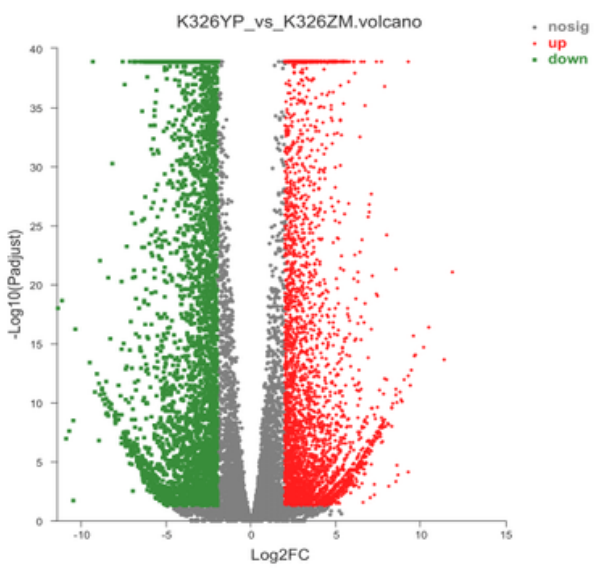

(c)
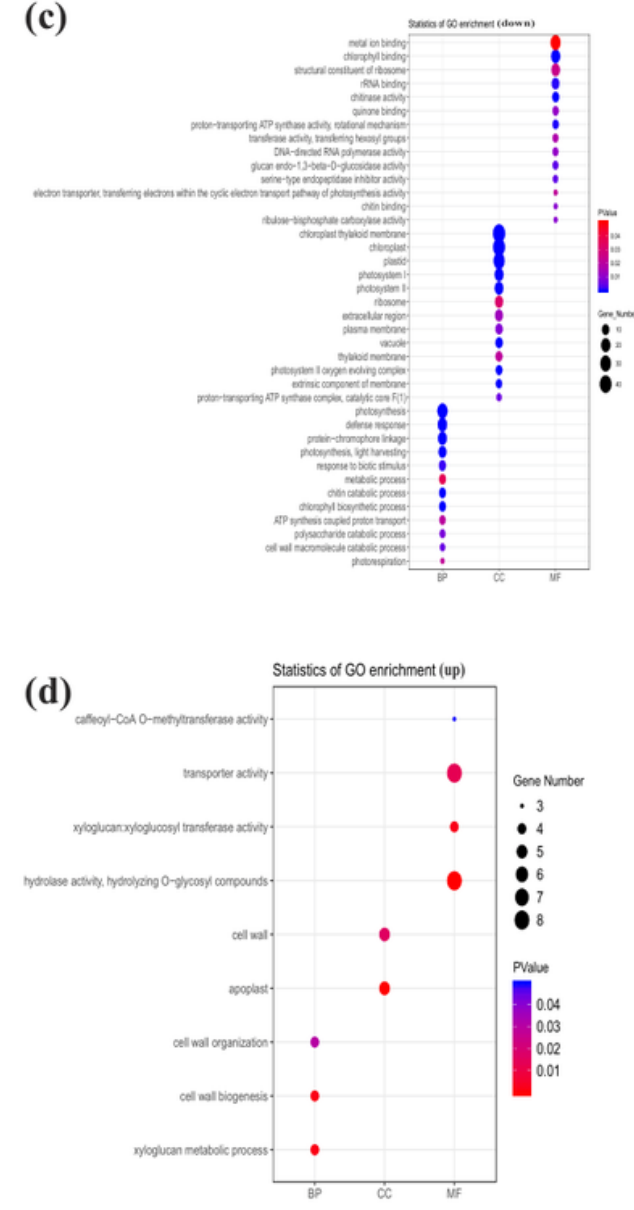

(e)
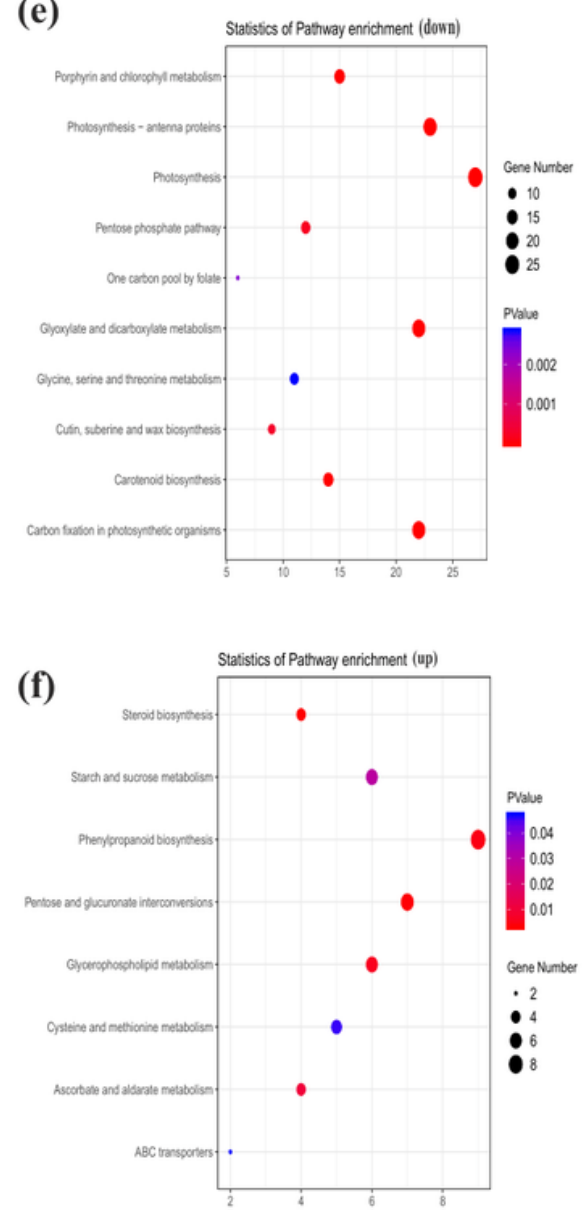

Figure 5

(a) The number of differentially expressed genes between lamina and midrib. (b) The volcano of differentially expressed genes between lamina and midrib. (c-d) Go enrichment of differentially expressed genes between lamina and midrib. (c-d) KEGG enrichment of differentially expressed genes between lamina and midrib. 
(a)

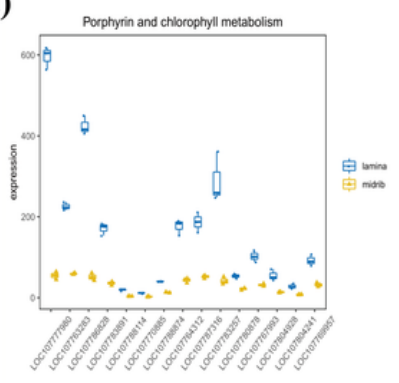

(b)

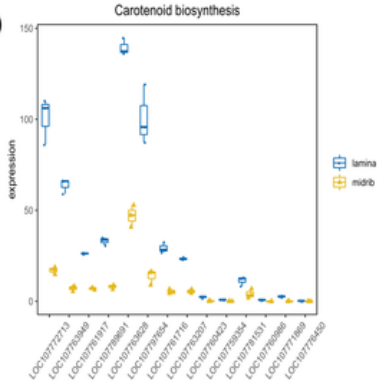

(c)

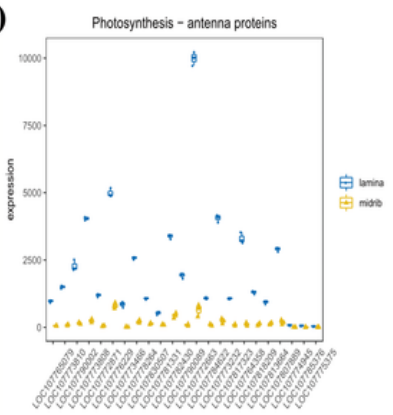

(d)

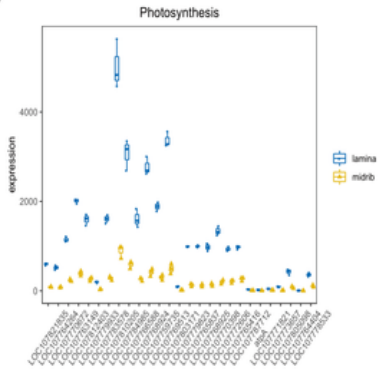

(e)

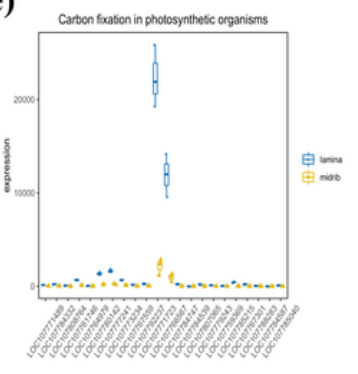

(f)

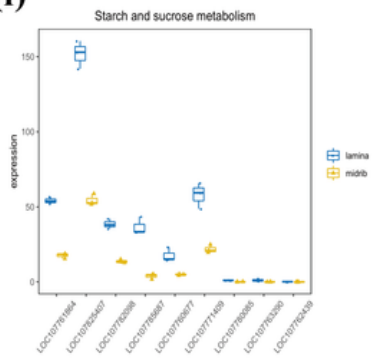

(g)

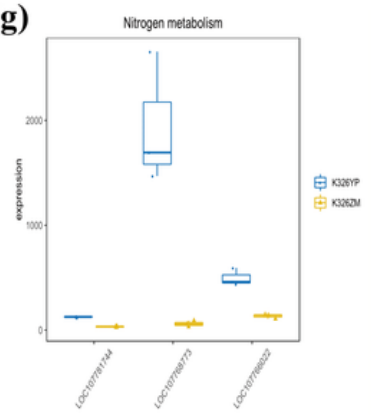

(h)

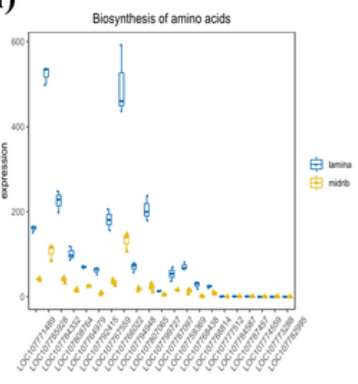

(i)

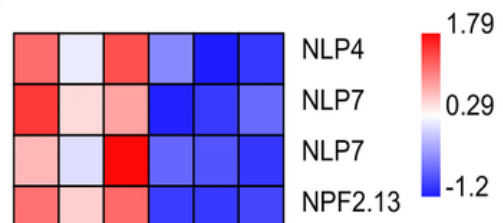

NPF2.13

NPF3.1

NPF3.1

NPF6.3

NPF6. 3

NPF7.3

NPF7.3

NPF7.3

NPF1.2

NPF1.2

NPF1.2

NPF1.2

NPF7.2

NPF7.2

NIA

NIA

GOGAT

GOGAT

GOGAT1

GOGAT

GS

GS

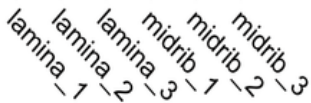

\section{Figure 6}

(a) Porphyrin and chlorophyll metabolism; (b) Carotenoid biosynthesis; (c) Photosynthesis-antenna proteins; (d) Photosynthesis; (e) Carbon fixation in photosynthetic organisms; (f) Sarch and sucrose metabolism; (g) Nitrogen metabolism; (h) Biosynthesis of amino acids; (i) Expression of genes involved in nitrate response, transport and assimilation. Box-whisker plot represents dispersity of minimum, first quartile, median, third quartile in genes expression level of treatments. Y-axis represents expression level. The columns represent six samples. The name of gene is on the right side,the up-or down-regulated proteins are indicated in red and green, respectively. The intensity of the colors increases with increasing expression level as noted on the color bar on the right side. 

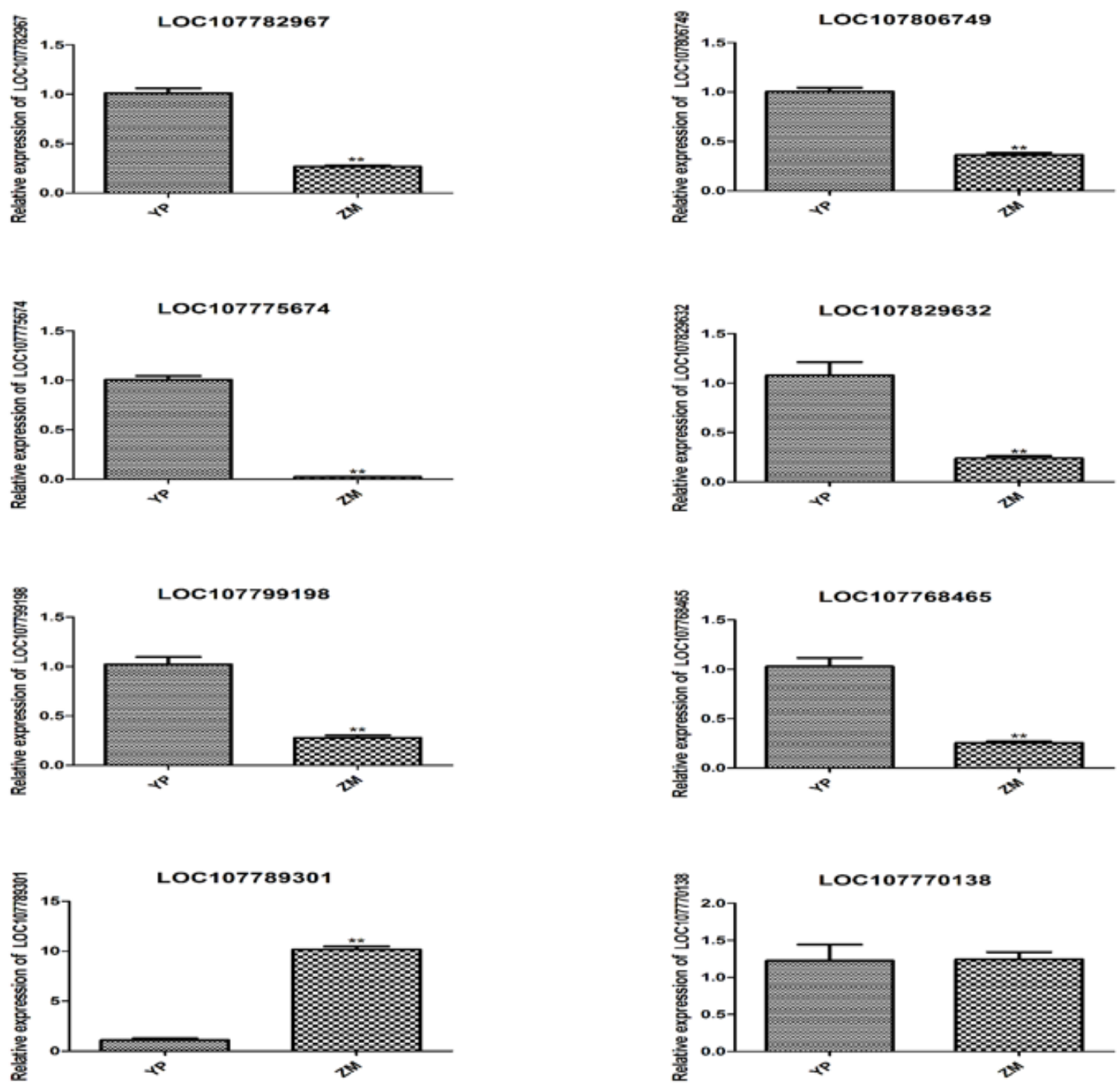

Figure 7

Expression levels of genes related to nitrate transport by qRT-PCR. The x-axis indicates the two samples. YP: Iamina of fluecured tobacco; ZM: midrib of flue-cured tobacco. The left y-axis indicated relative expression level of qRT-PCR. Error bars represent standard error of mean. 